\title{
Is an optical illusion the cause of classical bile duct injuries?
}

\author{
Francis Sutherland, MD \\ Chad G. Ball, MD \\ Jennifer Schendel, MD \\ Elijah Dixon, MD
}

Accepted Jan. 23, 2020

\section{Correspondence to: \\ F. Sutherland}

Foothills Hospital

1403 29th Street NW

Calgary AB T2N 2T9

francis.sutherland@

albertahealthservices.ca

DOI: $10.1503 /$ cjs.014019

\section{SUMmarY}

We sought to determine if lateral-inferior traction on the Hartmann pouch could produce bile duct kinking and subsequent misinterpretation of the space on the left side of the bile duct as the hepatobiliary triangle. Once traction was applied, we measured the angle between the cystic duct and inferior gallbladder wall (hepatobiliary triangle) in 76 cases, and the angle between the common bile duct and common hepatic duct (porta hepatis "triangle") in 41 cases. The mean angles were significantly different (hepatobiliary triangle mean $68.2^{\circ}$, standard deviation [SD] $16.0^{\circ}$, range $23-109^{\circ}$; porta hepatis "triangle" mean $112.0^{\circ}$, SD $18.4^{\circ}$, range $72-170^{\circ} ; p<0.01$ ). The ranges, however, overlapped in 26 cases. In many cases, lateral-inferior traction on the Hartmann pouch produced substantial kinking of the bile duct that could easily elicit the illusion that it is the hepatobiliary triangle rather than the centre of the porta hepatis.
B ile duct injuries often occur from a misinterpretation of anatomy. Way and Stewart recognized this in their video review of bile duct injuries, published in $2003 .{ }^{1}$ In a classical bile duct injury, the surgeon dissects the left side of the bile duct in the middle of the porta hepatis as if it were the hepatobiliary triangle. This produces a series of incorrect assumptions that eventually result in cutting the bile duct twice. ${ }^{2}$ Hugh and colleagues ${ }^{3}$ have also indicated that the bile duct may kink with traction on the Hartmann pouch, creating an optical illusion. This has never been previously confirmed in a formal manner.

The causes of bile duct injuries are multifactorial. Developing a "critical view of safety" ${ }^{4}$ is taught as the ultimate weapon against these injuries; however, they continue to occur. An illusion is defined as a scenario where an image or object is routinely misinterpreted, despite careful mental scrutiny. For surgeons, avoidance of an illusion can only be achieved by recognizing that in this particular circumstance, the operator's senses cannot be trusted. Can an illusion be a component in causing bile duct injuries?

The goal of our review was to determine if traction on the Hartmann pouch could cause the bile duct to kink, and therefore produce an angle in the middle of the porta hepatis that could be misinterpreted as the hepatobiliary triangle. We compared the angles created with traction on the Hartmann pouch in the hepatobiliary triangle and in the porta hepatis "triangle" from the images of 124 consecutive elective laparoscopic cholecystectomies. The images were initially obtained as part of a study documenting the regional anatomy landmarks used for navigation around the gallbladder (bile duct, sulcus of Rouviere, hepatic artery, umbilical fissure and enteric, also known as B-SAFE). ${ }^{5}$ Ethical review and approval for this study was provided by the health research ethics board of Alberta. The comparative analysis was completed with an unpaired $t$ test using SPSS version 19 (IBM).

Prior to dissection (and with lateral-inferior traction on the Hartmann pouch), we could measure the angle between the cystic duct and the inferior margin of the gallbladder (hepatobiliary triangle) in 76 out of 124 cases. 
Similarly, with traction, we could reliably measure an angle between the common bile duct and the common hepatic duct where the cystic duct inserts in 41 of 124 cases. Reliable measurement of this angle required an absence of adipose tissue and inflammation. The mean angle in the hepatobiliary triangle was $68.2^{\circ}$ (standard deviation [SD] $16.0^{\circ}$, range $23-109^{\circ}$ ) and the mean angle in the porta hepatis "triangle" was $112^{\circ}$ (SD $18.4^{\circ}$, range $\left.72-170^{\circ} ; p<0.01\right)$. We found that 26 of the 41 angles $(63 \%)$ measured within the porta hepatis overlapped with the range determined for the hepatobiliary triangle. We also identified that isolated elevation of the gallbladder often slightly kinked the bile duct, with a mean angle $164^{\circ}\left(180^{\circ}\right.$ indicates a straight bile duct). In a number of cases, traction produced a depressed cavity in the central porta hepatis, with

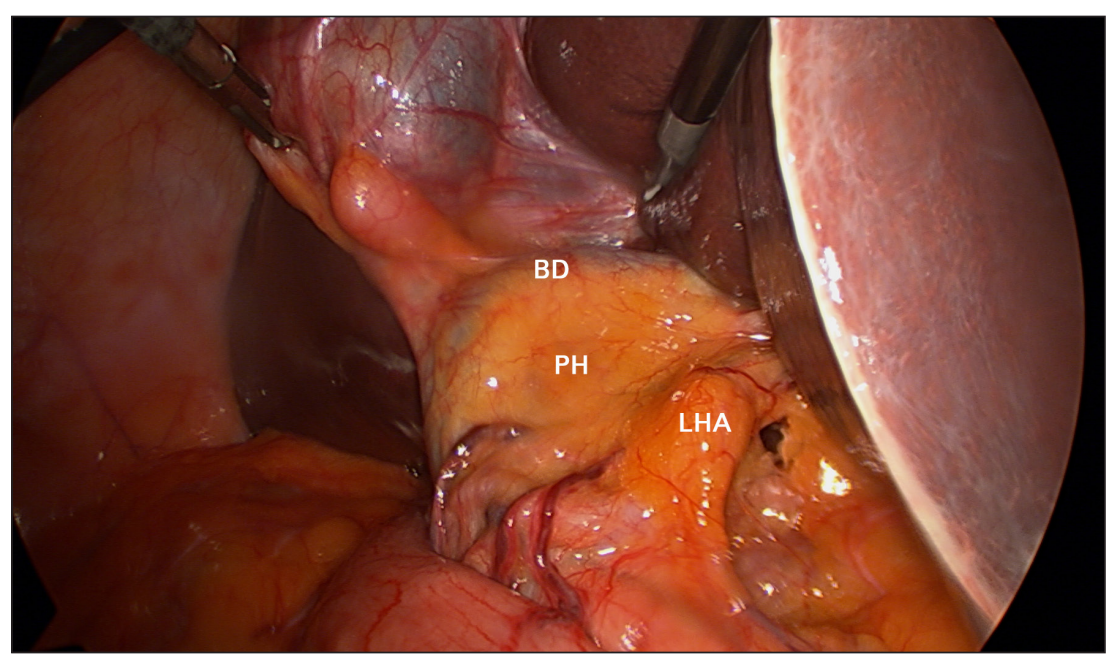

Fig. 1. Photograph of a laparoscopic cholecystectomy. In this procedure, traction on the Hartmann pouch has produced a $90^{\circ}$ kink in the bile duct. There is a cavity in the central porta hepatis $(\mathrm{PH})$ and a border created on the left side by the left hepatic artery (LHA). This space on the left side of the bile duct (BD) creates the illusion that it is the hepatobiliary triangle, seen above on the right side of the bile duct.

the left hepatic artery appearing as the bile duct. Four cases also displayed bifurcation of the left and right hepatic ducts within the porta hepatis, which appeared to increase the angle of the kink and hence the illusion.

In its native position, the extrahepatic bile duct is straight. During laparoscopic cholecystectomies, the gallbladder is elevated, with superior traction on the fundus. Subsequent lateral-inferior traction of the Hartmann pouch opens the hepatobiliary triangle. We have now shown that this traction does indeed kink the bile duct to a variable degree. In many cases, the kinking is quite profound (Fig. 1) and creates an illusion that this space is the hepatobiliary triangle. Dissection in this space may further increase kinking. Furthermore, a small right hepatic artery and lymph node are routinely found in this space, adding to this illusion. It should be noted that this illusion may appear at any time during dissection within the porta hepatis. In a patient with a low body mass index (BMI) and low inflammation, it may be present immediately and attract dissection into this plane. In a patient with a high BMI and high inflammation, the illusion may only become evident after much dissection in the central porta hepatis. The cognitive mistakes leading to bile duct injuries in these 2 circumstances may be different.

These data confirm that traction on the Hartmann pouch can produce kinking of the bile duct. This kinking may lead surgeons to misinterpret the central porta hepatis space (i.e., on the left side of the bile duct) as the hepatobiliary triangle. It is important that surgeons consider this hazard and not completely trust their visual senses, at the exclusion of all else, during hepatobiliary triangle dissection. In the context of acute or chronic inflammation of the hepatobiliary triangle or the gallbladder overlying the porta hepatis, the surgeons' attention may be further directed toward the central porta hepatis. We have previously indicated that using B-SAFE landmarks can prevent potentially faulty surgical navigation, and that an immediate correction is required to ensure a safe procedure..$^{5}$ We suggest that surgeons routinely take a moment to reorient themselves with these landmarks and look for a bile duct illusion before proceeding to divide the cystic duct.

Affiliations: From the Department of Surgery (Sutherland, Ball, Schendel, Dixon), University of Calgary, Calgary, Alta.

Competing interests: Chad G. Ball is coeditor-in-chief of $C F S$. He was not involved in the review or decision to accept this manuscript for publication. No other competing interests were declared.

Contributors: All authors contributed substantially to the conception, writing and revision of this article and approved the final version for publication.

Content licence: This is an Open Access article distributed in accordance with the terms of the Creative Commons Attribution (CC BYNC-ND 4.0) licence, which permits use, distribution and reproduction in any medium, provided that the original publication is properly cited, the use is noncommercial (i.e., research or educational use), and no modifications or adaptations are made. See: https://creativecommons. org/licenses/by-nc-nd/4.0/

\section{References}

1. Way LW, Stewart L, Gantert W, et al. Causes and prevention of laparoscopic bile duct injuries: analysis of 252 cases from a human factors and cognitive psychology perspective. Ann Surg 2003;237:460-9.

2. Sutherland F, Ball C. The heuristics and psychology of bile duct injuries. In: Dixon E, Vollmer CMJ, May GR, editors. Management of benign biliary stenosis and injury. New York: Springer; 2015:191-204.

3. Hugh TB. New strategies to prevent laparoscopic bile duct injury surgeons can learn from pilots. Surgery 2002;132:826-35.

4. Strasberg SM, Hertz M, Soper NJ. An analysis of the problems of biliary injury during laparoscopic cholecystectomy. 7 Am Coll Surg 1995;180:101-25.

5. Schendel J, Ball C, Dixon E, et al. Prevalence of anatomic landmarks for orientation during elective laparoscopic cholecystectomies. Surg Endosc 2020;34:3508-12. 